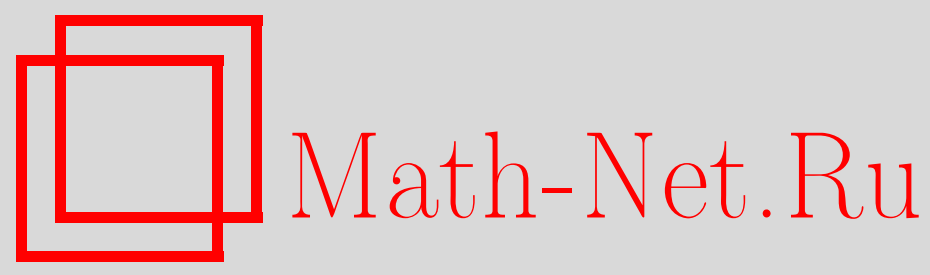

И. А. Чельцов, Регуляризация бирациональных автоморфизмов, Матем. заметки, 2004, том 76, выпуск 2, 286-299

DOI: https://doi.org/10.4213/mzm97

Использование Общероссийского математического портала Math-Net.Ru подразумевает, что вы прочитали и согласны с пользовательским соглашением http://www. mathnet.ru/rus/agreement

Параметры загрузки:

IP: 44.207 .124 .84

26 апреля 2023 г., 02:27:25

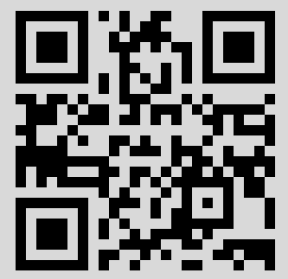




\title{
РЕГУЛЯРИЗАЦИЯ БИРАЦИОНАЛЬНЫХ АВТОМОРФИЗМОВ
}

И. А. Чельцов

\begin{abstract}
Рассмотрен вопрос об эффективной регуляризации бирациональных автоморфизмов многомерных алгебраических многообразий. Полученные методы явно применяются автором к нескольким трехмерньм многообразиям Фано и к поверхностям дель Пеццо над алгебраически незамкнутым полем.
\end{abstract}

Библиография: 10 названий.

1. Введение. В данной работе будет представлен эффективньй метод регуляризации конечных подгруп бирациональных автоморфизмов алгебраических поверхностей и трехмерных алгебраических многообразий. Полученный результат будет применен к описанию конечных подгруп бирациональных автоморфизмов гладкого двойного накрытия трехмерной квадрики, трехмерной квартики с одной обыкновенной двойной точкой, поверхностям дель Пецио степени 2 и 3 над алгебраически незамкнутьм полем (в частности, будет получен ответ на вопрос Манина, поставленный в книге [1]).

Всюду в статье все подразумевается проективным и определенньм над полем $\mathbb{F}$ характеристики нуль. Следующий результат хорошо известен.

ТеОрема 1.1. Пусть $X$ и - многообразия с каноническими особенностями $и$ обильными каноническими дивизорами. Тогда каждое бирачиональное отображение между $X$ и $Y$ является бирегулярным.

В частности, из теоремы 1.1 вытекает следующий результат.

СлЕДСТВИЕ 1.2. Пусть $X$ - многообразие с каноническими особенностями и обильным каноническим дивизором. Тогда каждый бирачиональный автоморфизм многообразия $X$ бирегулярен.

Из Программы минимальных моделей (см. [2]) вытекает следующий результат.

ТЕорема 1.3. Пусть $X$ - многообразие общего типа размерности 2 или 3. Тогда существует бирациональное отобрахение $\gamma: X \rightarrow V c$

$$
\gamma \circ \operatorname{Bir}(X) \circ \gamma^{-1}=\operatorname{Aut}(V)
$$

Легко видеть, что утверждение теоремы 1.3 не выполнено для многообразий с немаксимальной размерностью Кодаиры. 
ОПРЕДЕЛЕНИЕ 1.4. Для многообразия $X$ назовем подмножество $S$ групшы $\operatorname{Bir}(X)$ регуляризируемым на многообразии $V$ посредством бирачионального отображсения $\gamma: X \rightarrow V$, если

$$
\gamma \circ S \circ \gamma^{-1} \subset \operatorname{Aut}(V)
$$

а отображение $\gamma$ назовем регуляризачией множества $S \subset \operatorname{Bir}(X)$.

Каждое регуляризируемое подмножество групшы бирациональных автоморфизмов порождает регуляризируемую подгруппу, поэтому мы будем рассматривать в дальнейшем только регуляризируемые подгруппы.

Отметим, что регуляризация бирациональных автоморфизмов не определена единственньм образом и существуют бирациональные автоморфизмы, не обладающие регуляризацией. Следующий результат принадлежит И. Долгачеву.

УТВЕРЖДЕНИЕ 1.5. Достаточно общее бирачиональное квадратичное преобразование проективной плоскости нерегуляризируемо.

ДокАЗАТЕЛЬСтво. Рассмотрим достаточно общее бирациональное квадратичное преобразование $T: \mathbb{P}^{2} \rightarrow \mathbb{P}^{2}$. Можно считать, что порядок $T$ бесконечен и $T$ имеет три фундаментальные точки. Рассмотрим регуляризацию $f: X \rightarrow \mathbb{P}^{2}$ автоморфизма $T$ и бирегулярньй автоморфизм $g=f^{-1} \circ T \circ f$.

Предположим, что $x_{1}, \ldots, x_{n}$ - фундаментальные точки $f^{-1}$, не являющиеся фундаментальными точками $T$, и такие, что точки $T\left(x_{1}\right), \ldots, T\left(x_{n}\right)$ не являются фундаментальными точками отображения $T^{-1}$. Рассмотрим соответствующие исключительные кривые $E_{i}$.

Кривая $g\left(E_{i}\right)$ стягивается в точку $y_{i}=T\left(x_{i}\right)$ морфизмом $f$ и стягивается в точку $x_{j}$ морфизмом $f^{-1}$, поскольку по предположению точка $y_{i}$ не является фундаментальной точкой $T^{-1}$. Таким образом, $g$ переставляет кривые $E_{i}$ и некоторая степень $g^{N}$ отображения $g$ действует тривиально на кривых $E_{i}$. Следовательно, кривые $E_{i}$ могут быть стянуты, что даст такую регуляризацию $h: S \rightarrow \mathbb{P}^{2}$ бирационального автоморфизма $T^{N}$, что $h$ имеет не более чем 6 фундаментальных точек: три фундаментальные точки $T$ и три фундаментальные точки $T^{-1}$.

По построению бирегулярньй автоморфизм $h^{-1} \circ T^{N}$ о $h$ имеет бесконечньй порядок. $\mathrm{C}$ другой стороны, группа автоморфизмов поверхности, полученной раздутием от 4 до 8 точек на $\mathbb{P}^{2}$, конечна. Значит, $h$ является раздутием не более чем 3 точек и фундаментальные точки $T$ и $T^{-1}$ совпадают. С другой стороны, общее квадратичное преобразование таковым не является.

Заметим, что группа $\operatorname{Bir}\left(\mathbb{P}^{2}\right)$ порождена проективными автоморфизмами $\mathbb{P}^{2}$ и инволюциями Кремоны, которые все регуляризируемы.

СледСтвИЕ 1.6. Регуляризируемые әлементы группы бирачиональных автоморфизмов не обязательно образуют подгруппу.

Таким образом, для данного многообразия естественно попытаться описать регуляризируемые подгруппы групшы его бирациональных автоморфизмов. В общем случае такая проблема может быть очень сложной, однако хорошо известен следующий результат. 
Теорема 1.7. Пусть $X$ - алгебраическое многообразие, $а$ $G \subset \operatorname{Bir}(X)$ - конечная подгруппа. Тогда $G$ регуляризируема.

ДокАЗАтЕльство. Рассмотрим поле рациональных функций $K(X)$ на $X$, возьмем нормальную проективную модель $Y$ поля инвариантных функций $K(X)^{G}$ и рассмотрим нормализацию $V$ многообразия $Y$ в поле $K(X)$. В этом случае $V$ бирационально эквивалентно многообразию $X$ и группа $G$ действует бирегулярно на $V$.

Следует отметить, что доказательство теоремы 1.7 не очень эффективно - для данного многообразия и данной конечной подгрупш группы его бирациональных автоморфизмов найти ее регуляризацию достаточно трудно.

ЗАмЕЧАНИЕ 1.8. Основная цель данной работы - описать новьй и эффективный способ регуляризации конечных подгрупп бирациональных автоморфизмов поверхностей и трехмерных многообразий.

В п. 6 полученньй метод будет применен для нахождения регуляризации бирациональных инволюций двойного накрытия трехмерной квадрики.

Применение эквивариантного разрешения особенностей и применение Программы минимальных моделей дает следуюшее обобшение теоремы 1.7.

Теорема 1.9. Пусть $X$ - многообразие размерности 2 или 3, а $G$ - конечная подгруппа в $\operatorname{Bir}(X)$. Тогда существует бирачиональное отображсние $\gamma: X \rightarrow Y$ на многообразие $Y$ с терминальными особенностями такое, что либо канонический дивизор $K_{Y}$ численно әффективен, либо $Y$ обладает $\left(\gamma \circ G \circ \gamma^{-1}\right)$-эквивариантной структурой расслоения на многообразия Фано.

В дальнейшем мы применим эффективную регуляризацию к бирациональньм автоморфизмам бирационально жестких многообразий Фано.

ОПРЕДЕЛЕниЕ 1.10. Многообразие Фано $X$ назьвается бираиионально жестким, если $X$ имеет $\mathbb{Q}$-факториальные терминальные особенности, $\operatorname{Pic}(X)=\mathbb{Z}$ и $X$ бирационально не эквивалентен никакому расслоению Мори ${ }^{1}$, кроме самого себя.

Заметим, что из теоремы 1.9 вытекает следующее утверж дение.

Теорема 1.11. Пусть $X$ - бирационально жесткое многообразие Фано размерности не больше 3, а $G$ - конечная подгруппа в $\operatorname{Bir}(X)$. Тогда существует бираииональное отображсение $\gamma: X \rightarrow Y$ на многообразие Фано $Y$ с терминальными особенностями такое, что $\gamma \circ G \circ \gamma^{-1} \subset \operatorname{Aut}(Y)$.

Таким образом, теорема 1.11 дает способ нахождения всех конечных подгрупп группы бирациональных автоморфизмов бирационально жестких поверхностей дель Пецшо и трехмерных многообразий Фано. В п. 6 теорема 1.11 будет применена к двойному накрытию трехмерной квадрики, в п. 7 - к особой трехмерной квартике, а в п. 4 - к кубической поверхности в $\mathbb{P}^{3}$ и двойному накрытию $\mathbb{P}^{2}$.

Рассмотрим несколько открытых вопросов, связанных с регуляризацией бирациональных автоморфизмов.

\footnotetext{
${ }^{1}$ Расслоение $\tau: V \rightarrow Z$ называется расслоением Мори, если $\tau_{*}\left(\mathscr{O}_{V}\right)=\mathscr{O}_{Z}$, многообразие $V$ имеет терминальные $\mathbb{Q}$ факториальные особенности, дивизор $-K_{V}$ является $\tau$-обильным и $\operatorname{Pic}(V / Z)=\mathbb{Z}$.
} 
ГИПотеЗА 1.12. Группа бирациональных автоморфизмов порождена регуляризируемыми бирачиональными автоморфизмами.

Отметим, что подгруппа групш бирациональных автоморфизмов, порожденная регуляризируемьми бирациональными автоморфизмами, является нормальной.

ЗАмЕчаниЕ 1.13. Гипотеза 1.12 выполнена для $X$, если группа $\operatorname{Bir}(X)$ проста.

Утверждается гипотетически, что групшы $\operatorname{Bir}\left(\mathbb{P}^{2}\right)$ и $\operatorname{Bir}\left(\mathbb{P}^{3}\right)$ просты.

ГИПотеЗА 1.14. Для данного многообразия $X$ существуют порождающие груп$n$ вы $\operatorname{Bir}(X)$, которые регуляризируемы на многообразиях, лежсаших в конечном числе семейств.

Хорошо известно, что гипотезы 1.12 и 1.14 выполнены для поверхностей и для многих трехмерных бирационально жестких многообразий Фано (см. [3] и [4]).

2. Подвижные лог-пары. В этом пункте будут рассмотрены свойства подвижных лог-пар, которые были введены и рассмотрены в современном виде Алексеевым в работе [5]. Результаты этого пункта будут использованы в следующем пункте для конструкции алгоритма регуляризации.

ОПРЕДЕЛЕНИЕ 2.1. Под подвижной лог-парой

$$
\left(X, M_{X}\right)=\left(X, \sum_{i=1}^{n} b_{i} \mathscr{M}_{i}\right)
$$

будем подразумевать многообразие $X$ вместе с формальной конечной линейной комбинацией линейных систем $\mathscr{M}_{i}$ без неподвижных компонент таких, что все числа $b_{i}$ неотрицательны и рациональны.

Заметим, что каждая подвижная лог-пара может быть рассмотрена как обычная лог-пара. В частности, для подвижной лог-пары $\left(X, M_{X}\right)$ дивизор $K_{X}+M_{X}$ будет назьваться лог-каноническим дивизором лог-пары $\left(X, M_{X}\right)$, а $M_{X}$ будет называться граниией подвижной лог-пары $\left(X, M_{X}\right)$.

ЗАМЕчАнИЕ 2.2. Прямой образ границы подвижной лог-пары естественно определен для любого бирационального отображения.

Если лог-канонический дивизор подвижной лог-пары является $\mathbb{Q}-$ Картье дивизором, такие понятия как дискрепантность, терминальность и каноничность могут быть определены для подвижных лог-пар аналогично соответствуюшим определениям для обычных лог-пар.

ОПРЕДЕЛЕНИЕ 2.3. Скажем, что подвижные лог-пары $\left(X, M_{X}\right)$ и $\left(Y, M_{Y}\right)$ бupauиoнально әквивалентны, если существует бирациональное отображение $\rho: X \rightarrow Y$, такое что $M_{Y}=\rho\left(M_{X}\right)$.

Отметим, что любая подвижная лог-пара бирационально эквивалентна подвижной лог-паре с каноническими и терминальными особенностями. 
ОПРЕДЕЛЕНИЕ 2.4. Для данной подвижной лог-пары $\left(X, M_{X}\right)$ рассмотрим бирационально эквивалентную подвижную лог-пару $\left(W, M_{W}\right)$ с каноническими особенностями. Выберем такое $n \gg 0$, что дивизор

$$
D=n\left(K_{W}+M_{W}\right)
$$

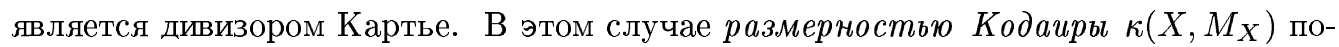
движной лог-пары $\left(X, M_{X}\right)$ будем назьвать размерность Иитаки лог-пары $(X, D)$.

Можно показать, что размерность Кодаиры подвижной лог-пары корректно определена и одинакова для всех бирационально эквивалентных лог-пар.

ЗАмечАниЕ 2.5. Размерность Кодаиры подвижной лог-пары является неубывающей функцией от коэффициентов ее границы.

Следующее определение совпадает с классическим в случае пустой границы.

ОПРЕДЕлЕниЕ 2.6. Скажем, что подвижная лог-пара $\left(V, M_{V}\right)$ является минимальной моделью подвижной лог-пары $\left(X, M_{X}\right)$, если они бирационально эквивалентны,

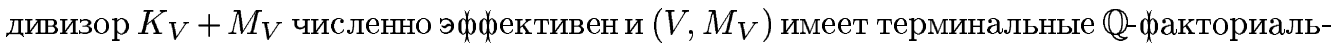
ные особенности.

Минимальная модель может не существовать и может не быть единственной. Однако минимальные модели важны в виду следующей стандартной гипотезы.

ГИПоТЕЗА 2.7. Пусть $\left(X, M_{X}\right)$ - произвольная подвижная лог-пара. Тогда либо ее размерность Кодаиры неотрицательна $и\left(X, M_{X}\right)$ бирачионально әквивалентна минимальной модели, либо $\kappa\left(X, M_{X}\right)=-\infty$ и подвижная лог-пара $\left(X, M_{X}\right)$ бирационально әквивалентна подвижнной лог-паре $\left(Y, M_{Y}\right)$ с $\mathbb{Q}$-факториальньми терминальными особенностями такой, что $Y$ имеет структуру расслоения Морu $f: Y \rightarrow Z$, а дивизор $-\left(K_{Y}+M_{Y}\right)$ является $f$-обильным.

УТВЕРЖДЕНИЕ 2.8. Гипотеза 2.7 выполнена в размерности 2 и 3.

Рассмотрим морфизм, связанньй с минимальной моделью подвижной лог-пары.

ОПРЕДЕЛЕНИЕ 2.9. Предположим, что подвижная лог-пара $\left(X, M_{X}\right)$ является минимальной моделью. Тогда морфизм $I\left(X, M_{X}\right): X \rightarrow Z$ будем назьвать морфизмом Иитаки подвижной лог-пары $\left(X, M_{X}\right)$, если для некоторого обильного $\mathbb{Q}$-Картье дивизора $L$ на многообразии $Z$ вьполнено

$$
K_{X}+M_{X} \sim_{\mathbb{Q}} I\left(X, M_{X}\right)^{*}(L)
$$

и $I\left(X, M_{X}\right)_{*}\left(\mathscr{O}_{X}\right)=\mathscr{O}_{Z}$.

Что можно сказать о существовании морфизма Иитаки?

ГИПотеЗА 2.10. Морфизм Иитаки существует.

Отметим, что стандартная гипотеза о лог-избыточности (см. [2]) влечет гипотезу 2.10. В частности, гипотеза 2.10 вьполнена для всех поверхностей и для всех трехмерных многообразий (см., например, [6]). 
УТВЕРЖДЕНИЕ 2.11. Пусть $X$ - алгебраическая поверхность или трехмерное многообразие, $а\left(X, M_{X}\right)$ - подвижная лог-пара. В этом случае морфизм Иитаки минимальной модели подвижной лог-пары $\left(X, M_{X}\right)$ существует.

Как уже упоминалось, минимальная модель подвижной лог-пары может не быть единственной. Однако, следующее рациональное отображение всегда единственно.

ОПРЕДЕЛЕНИЕ 2.12. Предположим, что подвижная лог-пара $\left(X, M_{X}\right)$ бирационально эквивалентна минимальной модели $\left(V, M_{V}\right)$ посредством бирационального отображения $\rho: X \rightarrow V$, и предположим, что морфизм Иитаки подвижной лог-пары $\left(V, M_{V}\right)$ существует. Тогда будем называть отображение

$$
I\left(X, M_{X}\right)=I\left(V, M_{V}\right) \circ \rho
$$

отображением Иитаки подвижной лог-пары $\left(X, M_{X}\right)$.

Можно показать, что в определении 2.12 отображение Иитаки подвижной лог-пары не зависит от выбора минимальной модели. Следующее определение является непосредственньм обобщением классической канонической модели.

ОпРЕДЕЛЕниЕ 2.13. Будем говорить, что подвижная лог-пара $\left(V, M_{V}\right)$ является канонической моделью подвижной лог-пары $\left(X, M_{X}\right)$ в том случае, когда они бирационально эквивалентны, дивизор $K_{V}+M_{V}$ обилен и особенности $\left(V, M_{V}\right)$ каноничны.

Следующий результат доказьвается аналогично соответствующему утверждению для обычных лог-пар (см. [6]).

Теорема 2.14. Каноническая модель единственна, если она существует.

Из теоремы 2.14 следует простой, но важный результат.

СледСтвиЕ 2.15. Предположим, что $\left(X, M_{X}\right)$ - каноническая модель. Тогда все бирачиональные автоморфизмы $X$, сохраняющие подвижную лог-пару $\left(X, M_{X}\right)$, бирегулярны.

Отметим, что если каноническая модель подвижной лог-пары $\left(X, M_{X}\right)$ существует, то $\kappa\left(X, M_{X}\right)$ равно размерности многообразия $X$. Более того, легко видеть, что с точностью до гипотезы 2.7 выполнено и обратное (см. [2]).

3. Эффективная регуляризация. В этом пункте будет описан эффективный алгоритм нахождения регуляризации конечной подгруппы групшы бирациональных автоморфизмов.

Пусть $X$ - поверхность или трехмерное многообразие, а $G$ - конечная подгрупша в $\operatorname{Bir}(X)$. Зафиксируем некоторьй очень обильный дивизор $H$ на $X$ вместе с неотрищательным рациональным числом $\mu$ и положим

$$
\left(X, H_{X}^{\mu}\right)=\left(X, \sum_{g \in G} \mu g(|H|)\right) .
$$

По построению, подвижная лог-пара $\left(X, H_{X}^{\mu}\right)$ является $G$-инвариантной. 
Лемма 3.1. Имеет место равенство $\kappa\left(X, H_{X}^{\mu}\right)=\operatorname{dim}(X) \partial_{\text {ля }} \mu \gg 0$.

ДокАЗАТЕльство. Размерность Кодаиры подвижной лог-пары является неубьвающей функцией от коэффициентов ее границы. Таким образом,

$$
\kappa\left(X, H_{X}^{\mu}\right) \geqslant \kappa(X, \mu|H|) .
$$

Из обильности $H$ следует, что $\kappa(X, \mu|H|)=\operatorname{dim}(X)$ для $\mu \gg 0$.

Итак, $\kappa\left(X, H_{X}^{\mu}\right)=\operatorname{dim}(X)$ для достаточно больших $\mu$ по лемме 3.1. Следовательно, утверждения 2.8 и 2.11 дают бирациональную перестройку $\sigma: X \rightarrow-$ такую, что подвижная лог-пара

$$
\left(V, H_{V}^{\mu}\right)=\left(\sigma(X), \sigma\left(H_{X}^{\mu}\right)\right)
$$

является канонической моделью подвижной лог-пары $\left(X, H_{X}^{\mu}\right)$.

Лемма 3.2. Рациональное отображение $\sigma$-регуляризачия подгруппы $G$.

ДокАЗАтЕльство. Подвижная лог-пара $\left(V, H_{V}^{\mu}\right)$ является $\left(\sigma \circ G \circ \sigma^{-1}\right)$-инвариантной канонической моделью. Необходимое утверждение вытекает из следствия 2.15.

Таким образом, эффективно получена регуляризация группы $G$. В частности, получено эффективное доказательство теоремы 1.7 для поверхностей и трехмерных многообразий. Более того, принимая во внимание $G$-инвариантное разрешение особенностей и Лог-программу минимальных моделей, получаем, что последнее дает эффективную регуляризацию подгруппы $G$, удовлетворяющую всем требованиям теоремы 1.9 .

Покажем теперь, что особенности самого многообразия $V$ также канонические.

\section{Лемма 3.3. Особенности $V$ каноничны.}

ДоКАЗАТЕЛЬСТВо. Из Второй главной теоремы работы [6] следует, что для некоторого рационального числа $\delta>\mu$ каноническая модель подвижной лог-пары $\left(X, H_{X}^{\delta}\right)$ является $\left(V, H_{V}^{\delta}\right)$. В частности, оба лог-канонических дивизора

$$
K_{V}+H_{V}^{\mu} \quad \text { и } \quad K_{V}+H_{V}^{\delta}
$$

являются дивизорами $\mathbb{Q}-$ Картье. Значит, канонический дивизор

$$
K_{V} \sim_{\mathbb{Q}}\left(K_{V}+H_{V}^{\mu}\right)-\frac{\mu}{\delta-\mu}\left[\left(K_{V}+H_{V}^{\delta}\right)-\left(K_{V}+H_{V}^{\mu}\right)\right]
$$

также является дивизором $\mathbb{Q}-$ Картье.

Подвижная лог-пара $\left(V, H_{V}^{\mu}\right)$ является канонической моделью. В частности, особенности подвижной лог-пары $\left(V, H_{V}^{\mu}\right)$ каноничны. С другой стороны, каноничность особенностей $V$ следует из каноничности особенностей подвижной лог-пары $\left(V, H_{V}^{\mu}\right)$ и уже доказанного факта, что дивизор $K_{V}$ является дивизором $\mathbb{Q}-$ Картье.

4. Поверхности дель Пеццо. В этом пункте мы рассмотрим поверхности дель Пещо над алгебраически незамкнутым полем в контексте регуляризации бирациональных автоморфизмов.

Зафиксируем гладкую поверхность дель Пещц $X$ с $\operatorname{Pic}(X)=\mathbb{Z}$ и $K_{X}^{2}$ равным 2 или 3 . Отметим, что из условия $\operatorname{Pic}(X)=\mathbb{Z}$ следует, что поле определения $\mathbb{F}$ не является алгебраически замкнутым. 
ЗАмЕЧАнИЕ 4.1. Хорошо известно, что

$X \cong\left\{\begin{array}{l}\text { двойное накрытие } \mathbb{P}^{2}, \text { разветвленное в гладкой квартике, если } K_{X}^{2}=2, \\ \text { кубика в } \mathbb{P}^{3}, \text { если } K_{X}^{2}=3 .\end{array}\right.$

Напомним коротко конструкцию инволюций Бертини и Гейзера поверхности $X$. Рассмотрим бирациональный морфизм $f: W \rightarrow X$ такой, что поверхность $W$ неособа и $K_{W}^{2}>0$. Из условия $\operatorname{Pic}(X)=\mathbb{Z}$ следует, что антиканонический дивизор $-K_{W}$ численно эффективен и объемен. Следовательно, линейная система $\left|-n K_{W}\right|$ свободна для $n \gg 0$ и задает бирациональньй морфизм на поверхность дель Пеццо $V$ с каноническими особенностями степени 1 или 2.

ОПРЕДЕЛЕниЕ 4.2. Скажем, что бирациональное отображение $\phi_{\mid-n K_{W}} \circ f^{-1}$ является стандартным отображением поверхности дель Пеццо $X$ в поверхность дель Пеццо $V$.

Можно показать, что в случае $K_{V}^{2}=1$ поверхность $V$ является двойньм накрытием квадратичного конуса в $\mathbb{P}^{3}$ с ветвлением в квартике, а в случае $K_{V}^{2}=2$ поверхность $V$ является двойным накрытием $\mathbb{P}^{2}$ с ветвлением в квартике. Таким образом, $V$ обладает канонической бирегулярной инволюцией, которая индуцирует бирациональную инволюцию $\tau$ поверхности дель Пеццо $X$. В случае, когда поверхность $V$ гладкая, инволюция $\tau$ не бирегулярна и $\tau$ назьвается инволюиией Бертини в случае $K_{V}^{2}=1$ или инволючией Гейзера в случае $K_{V}^{2}=2$. Классические и более геометричные определения инволюций Бертини и Гейзера можно найти в работах [7] и [8].

ЗАмЕчаниЕ 4.3. Поверхность $V$ гладкая тогда и только тогда, когда $V \cong W$.

Важность и сущность инволюций Бертини и Гейзера выражена в следующем классическом результате Манина (см. [7] и [8]).

Теорема 4.4. Поверхность $X$ бирационально жесткая, а группа $\operatorname{Bir}(X)$ является полупрямым продуктом группы $\mathrm{Aut}(X)$ и подгруппы, порожденной всеми инволюииями Бертини и Гейзера поверхности $X$.

В работе [9] был доказан следующий результат.

Теорема 4.5. Предположим, что $Y$ - поверхность дель Пецио с каноническими особенностями, бирационально әквивалентная поверхности $X$. Тогда $Y$ мохет быть получена стандартным преобразованием поверхности $X$.

Отметим, что из теорем 4.5 и 1.11 вытекает следующее.

УТВЕРЖДЕНИЕ 4.6. Каждая конечная подгруппа группы $\operatorname{Bir}(X)$ является либо подәруппой грyпnы $\operatorname{Aut}(X)$, либо подәруппой групnы $\operatorname{Aut}(V)$, где $V$ - гладкая поверхность дель Пецио, полученная стандартным преобразованием из поверхности $X$.

5. Вопрос Ю. И. Манина. В этом пункте мы ответим на вопрос Манина, поставленный в $[1]$.

Пусть $X$ - гладкая кубическая поверхность в $\mathbb{P}^{3}$ с $\operatorname{Pic}(X)=\mathbb{Z}$ над алгебраически незамкнутым полем $\mathbb{F}$, а $G$ - группа бирациональных автоморфизмов поверхности $X$, порожденная всеми инволюциями Бертини и инволюциями Гейзера поверхности $X$. 
ЗАмЕчАНИЕ 5.1. Из построения инволюций Бертини и Гейзера следует, что $G$ является нормальной подгруппой в групе $\operatorname{Bir}(X)$.

Следующий результат является специальным случаем теоремы 4.4.

ТЕОРема 5.2. Кубика $X$ бирационально жестка, а әруппа $\operatorname{Bir}(X)$ является полупрямым произведением группы $G$ и группь $\operatorname{Aut}(X)$.

В работе [1] был поставлен вопрос о том, какие элементы груп $G$ и $\operatorname{Bir}(X)$ имеют конечньй порядок. Отметим, что конечный порядок имеют все бирегулярные автоморфизмы кубики $X$, инволюции Бертини и Гейзера, а также все сопряженные им элементы группы $\operatorname{Bir}(X)$.

ЗАмЕчАниЕ 5.3. Пусть $\theta$ - бирегулярный автоморфизм кубики $X$, а $\tau$ - бирациональная инволюция Бертини или бирациональная инволюция Гейзера кубики $X$ такая, что $\theta \circ \tau=\tau \circ \theta$. Тогда $\tau \circ \theta$ имеет конечньй порядок.

Несколько позднее, Каневский описал в работе [10] элементы групшы $G$, которые имеют конечный порядок. Отметим, что его метод чисто теоретико-групшовой и основан на образуюших соотношениях в группе $G$, найденных в [8].

ТЕорема 5.4. Каждый бирациональный автоморфизм кубики $X$ конечного порядка сопряжен в группе $\operatorname{Bir}(X)$ одному из следующ, бх бирациональных автоморфизмов:

а) бирегулярный автоморфизм кубики $X$;

б) инволюиия Бертини;

в) инволюиия Гейзера;

г) композичия коммутирующих между собой бирегулярного автоморфизма кубической поверхности $X$ и инволючии Бертини или Гейзера.

Отметим, что теоремы 5.2 и 5.4 влекут следующее

СлЕДСТВИЕ 5.5. Элементы конечного порядка в әруппе $G$ сопряжены с инволюииями Бертини и Гейзера.

ДОКАЗАТЕЛЬСТВО ТЕОРЕМЫ 5.4. Пусть $\tau$ - элемент конечного порядка в $\operatorname{Bir}(X)$. Необходимо показать, что $\tau$ сопряжен одному из следующих бирациональных автоморфизмов: бирегулярньй автоморфизм кубики $X$; инволюция Бертини; инволюция Гейзера; композищия коммутирующих между собой бирегулярного автоморфизма кубики $X$ и инволюции Бертини или Гейзера.

Из бирациональной жесткости кубики $X$ и теоремы 1.11 следует, что бирациональный автоморфизм $\tau$ может быть регуляризован на некоторой гладкой поверхности дель Пещи $Y$. Таким образом, существует бирациональное отображение $\gamma: X \rightarrow Y$ такое, что $\gamma \circ \tau \circ \gamma^{-1} \in \operatorname{Aut}(Y)$. С другой стороны, по теореме 4.5 поверхность $Y$ бирегулярна либо самой кубике $X$, либо раздутию кубики $X$. В первом случае $\tau$ сопряжен бирегулярному автоморфизму поверхности $X$. Можно считать, что существует раздутие $f: Y \rightarrow X$ и $K_{Y}^{2}=1$ или $K_{Y}^{2}=2$.

Предположим, что $K_{Y}^{2}=2$. Тогда

$$
\operatorname{Pic}(Y)=\mathbb{Z} K_{Y} \oplus \mathbb{Z} E
$$

где $E$ - исключительный дивизор раздутия $f$. 
Отметим, что действие $\gamma \circ \tau$ о $\gamma^{-1}$ на $\operatorname{Pic}(Y)$ должно сохранять класс $K_{Y}$. Если

$$
\gamma \circ \tau \circ \gamma^{-1}(E) \sim E
$$

то отображение $f \circ \gamma \circ \tau \circ \gamma^{-1} \circ f^{-1}$ бирегулярно. Таким образом, можно считать, что кривая $E$ не $\left(\gamma \circ \tau \circ \gamma^{-1}\right)$-инвариантна. Непосредственные вычисления дают

$$
\left(\gamma \circ \tau \circ \gamma^{-1}\right)^{*}\left(K_{X}\right) \sim 2 f^{*}\left(K_{X}\right)+3 E, \quad\left(\gamma \circ \tau \circ \gamma^{-1}\right)^{*}(E) \sim-f^{*}\left(K_{X}\right)-2 E .
$$

Из конструкции инволюции Гейзера следует, что для некоторой инволюции Гейзера $\sigma$ кубической поверхности $X$ действие

$$
\gamma \circ \tau \circ \gamma^{-1} \circ f^{-1} \circ \sigma \circ f
$$

на групе $\operatorname{Pic}(Y)$ тривиально. Значит, отображение $f \circ \gamma \circ \tau \circ \gamma^{-1} \circ f^{-1} \circ \sigma$ является бирегулярньм автоморфизмом кубики $X$, который, к тому же, сохраняет точку $f(E)$, откуда следует, что $f \circ \gamma \circ \tau \circ \gamma^{-1} \circ f^{-1} \circ \sigma$ коммутирует с инволюцией $\sigma$.

Предположим теперь, что $K_{Y}^{2}=1$ и

$$
\operatorname{Pic}(Y)=\mathbb{Z} K_{Y} \oplus \mathbb{Z} E,
$$

где $E$ - исключительный дивизор бирационального морфизма $f$.

Можно считать, что действие $\gamma \circ \tau \circ \gamma^{-1}$ на $\operatorname{Pic}(Y)$ нетривиально, поскольку в противном случае бирациональный автоморфизм $f \circ \gamma \circ \tau \circ \gamma^{-1} \circ f^{-1}$ бирегулярен. Непосредственные вычисления показьвают, что

$$
\left(\gamma \circ \tau \circ \gamma^{-1}\right)^{*}\left(K_{X}\right) \sim 5 f^{*}\left(K_{X}\right)+6 E, \quad\left(\gamma \circ \tau \circ \gamma^{-1}\right)^{*}(E) \sim-4 f^{*}\left(K_{X}\right)-5 E .
$$

Из конструкции инволюции Бертини следует, что для некоторой инволюции Бертини $\sigma$ кубики $X$ композиция $f \circ \gamma \circ \tau \circ \gamma^{-1} \circ f^{-1} \circ \sigma$ бирегулярна и сохраняет $f(E)$, откуда следует, что автоморфизм $f \circ \gamma \circ \tau \circ \gamma^{-1} \circ f^{-1} \circ \sigma$ коммутирует с $\sigma$.

Теперь рассмотрим оставшийся случай: $K_{Y}^{2}=1$ и

$$
\operatorname{Pic}(Y)=\mathbb{Z} K_{Y} \oplus \mathbb{Z} E_{1} \oplus \mathbb{Z} E_{2},
$$

где $E_{1}$ и $E_{2}$ - две различные исключительные кривые морфизма $f$.

Как и в двух предыдущих случаях можно считать, что действие $\gamma \circ \tau \circ \gamma^{-1}$ на группе $\operatorname{Pic}(Y)$ нетривиально. По аналогичным причинам можно считать, что

$$
\gamma \circ \tau \circ \gamma^{-1}\left(E_{1} \cup E_{2}\right) \neq E_{1} \cup E_{2}
$$

Предположим, что кривая $E_{1}$ является $\left(\gamma \circ \tau \circ \gamma^{-1}\right)$-инвариантной. В этом случае положим $g: Y \rightarrow V$ - сдутие кривой $E_{1}$. Тогда $g \circ \gamma \circ \tau \circ \gamma^{-1} \circ g^{-1}$ является бирегулярным автоморфизмом поверхности $V$. С другой стороны, поверхность $V$ является гладкой поверхностью дель Пещо с $K_{V}^{2}=2$. Теперь, как и в случае, когда $K_{Y}^{2}=2$, несложно показать, что автоморфизм $f \circ \gamma \circ \tau \circ \gamma^{-1} \circ f^{-1}$ является композицией инволюции Гейзера кубики $X$ и коммутирующего бирегулярного автоморфизма кубики $X$.

Можно считать, что ни одна из кривых $E_{i}$ не является $\left(\gamma \circ \tau \circ \gamma^{-1}\right)$-инвариантной, откуда следует, что

$$
\begin{aligned}
\left(\gamma \circ \tau \circ \gamma^{-1}\right)^{*}\left(K_{X}\right) & \sim 5 f^{*}\left(K_{X}\right)+6 E_{1}+6 E_{2}, \\
\left(\gamma \circ \tau \circ \gamma^{-1}\right)^{*}\left(E_{1}\right) & \sim-2 f^{*}\left(K_{X}\right)-2 E_{1}-3 E_{2}, \\
\left(\gamma \circ \tau \circ \gamma^{-1}\right)^{*}\left(E_{2}\right) & \sim-2 f^{*}\left(K_{X}\right)-3 E_{1}-2 E_{2} .
\end{aligned}
$$

Из конструкции инволюции Бертини следует, что $f \circ \gamma \circ \tau \circ \gamma^{-1} \circ f^{-1}$ является композицией коммутирующих инволюции Бертини кубики $X$ и бирегулярного автоморфизма кубики $X$. 
6. Двойное накрытие квадрики. В этом пункте мы эффективно применим алгоритм регуляризации, полученньй в пункте 3 .

Зафиксируем двойное накрытие

$$
\theta: X \rightarrow Q \subset \mathbb{P}^{4}
$$

гладкой трехмерной квадрики $Q$, разветвленное в гладкой октике $S \subset Q$. Многообразие $X$ является многообразием $\Phi$ ано с $\operatorname{Pic}(X) \cong \mathbb{Z}$ и $-K_{X}^{3}=4$. Более того, $X$ бирационально жестко (см. [3]).

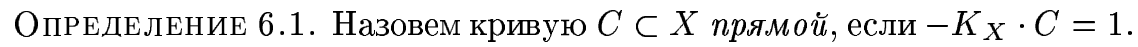

Многообразие $X$ содержит одномерное семейство прямых, если $\mathbb{F}=\mathbb{C}$. Каждая прямая $C$ на $X$ индуцирует бирациональную инволюцию $\tau_{C}$ в $\operatorname{Bir}(X)$ такую, что $\tau_{C}$ небирегулярна в том и только том случае, когда $\theta(C) \not \subset S$ (см. [3]). Сущность инволюций $\tau_{C}$ заключена в следующем результате Исковских (см. [3]).

Теорема 6.2. Группа $\operatorname{Bir}(X)$ является полупрямым произведением группы автоморфизмов трехмерного многообразия $X$ и нормальной подгруппы, порожденной небирегулярными инволюииями $\tau_{C}$.

Инволюции $\tau_{C}$ регуляризируемы по теореме 1.7. Значит, из теоремы 6.2 следует, что гипотезы 1.12 и 1.14 вьполнены для многообразия $X$.

Зафиксируем прямую $C$ на $X$ такую, что $\theta(C)$ не содержится в поверхности $S$. Тогда бирациональная инволюция $\tau_{C}$ не бирегулярна и из теоремы 1.11 следует существование бирационального отображения $\gamma: X \rightarrow X_{C}$ такого, что $X_{C}$ - трехмерное многообразие $\Phi$ ано с терминальными особенностями и

$$
\gamma \circ \tau_{C} \circ \gamma^{-1} \in \operatorname{Aut}\left(X_{C}\right)
$$

Теперь покажем, как явно построить бирациональное отображение $\gamma$, используя полученньй алгоритм регуляризации. Для некоторого рационального числа $\mu>0$ рассмотрим подвижную лог-пару

$$
\left(X, H_{X}^{\mu}\right)=\left(X, \mu\left|-K_{X}\right|+\mu \tau_{C}\left(\left|-K_{X}\right|\right)\right) .
$$

ЛЕмма 6.3. Особенности подвижной лог-пары $\left(X, H_{X}^{\mu}\right)$ терминальны для $\mu<$ $1 / 10$, каноничны для $\mu=1 / 10$ и неканоничны для $\mu>1 / 10$. Более того,

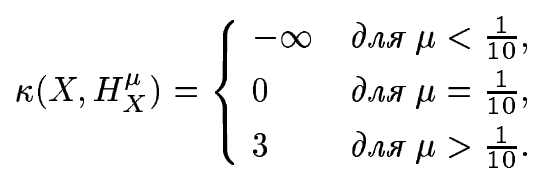


ДокАЗАТЕльство. Все утверждения получаются с помощью легких вычислений, основанных на непосредственной конструкции бирациональной инволюции $\tau_{C}$, приведенной в работе [3].

Значит, по следствию 2.15 отображение $I\left(X, H_{X}^{\mu}\right)$ является регуляризацией бирациональной инволюции $\tau_{C}$ для $\mu>1 / 10$. Опишем явно отображение Иитаки $I\left(X, H_{X}^{\mu}\right)$ для $\mu>1 / 10$. Раздуем $f: W \rightarrow X$ прямую $C$. Пусть $Z$ - единственная базисная кривая линейной системы $\left|-K_{W}\right|$. Раздуем $g: V \rightarrow W$ кривую $Z$, положим $G=g^{-1}(Z)$ и рассмотрим подвижную лог-пару

$$
\left(V, H_{V}^{\mu}\right)=\left(V,(f \circ g)^{-1}\left(H_{X}^{\mu}\right)\right) .
$$

ЗАмЕчаниЕ 6.4. Подвижная лог-пара $\left(V, H_{V}^{\mu}\right)$ терминальна для всех $\mu>0$.

Значит, можно применить Лог-программу минимальных моделей к $\left(V, H_{V}^{\mu}\right)$ и получить для $\mu>1 / 10$ бирациональное отображение $\rho: V \rightarrow Y$ такое, что подвижная лог-пара $\left(Y, H_{Y}^{\mu}\right)=\left(Y, \rho\left(H_{V}^{\mu}\right)\right)$ имеет терминальные $\mathbb{Q}$-факториальные особенности, а дивизор $K_{Y}+H_{Y}^{\mu}$ численно эффективен и объемен.

ЗАмЕчАниЕ 6.5. Явная конструкция отображения $\rho$ описана в работе [9], где показано, что $\rho$ является композицией флопа в некоторой кривой $T$, содержашейся в дивизоpe $G$, а стягиванием собственного образа дивизора $G$ в циклическую фактор-особенность типа $\frac{1}{2}(1,1,1)$.

Для $\mu=1 / 10$ бирациональный морфизм $f: W \rightarrow X$ является крепантньм по отношению к подвижной лог-паре $\left(X, H_{X}^{\mu}\right)$. Значит, $K_{Y}+H_{Y}^{\mu} \sim_{\mathbb{Q}} 0$ для $\mu=1 / 10$ и, следовательно, антиканонический дивизор $-K_{Y}$ является численно эффективным и объемным. Теперь можно применить утверждение 2.11 к подвижной лог-паре $\left(Y, H_{Y}^{\mu}\right)$ для $\mu>1 / 10$ и получить бирациональный морфизм

$$
I\left(Y, H_{Y}^{\mu}\right)=\psi_{\left|-n\left(K_{Y}+H_{Y}^{\mu}\right)\right|} \quad \text { для некоторого } n \gg 0 .
$$

По построению $X_{C}=I\left(Y, H_{Y}^{\mu}\right)(Y)$ является многообразием Фано с каноническими особенностями. Более того, можно показать, что бирациональньй морфизм $I\left(Y, H_{Y}^{\mu}\right)$ не стягивает дивизоров на многообразии $Y$. Таким образом, особенности многообразия $X_{C}$ терминальны, но легко видеть, что они не $\mathbb{Q}$-факториальны. Следовательно, для $\mu>1 / 10$ бирациональное отображение

$$
\gamma=I\left(Y, H_{Y}^{\mu}\right) \circ \rho \circ(f \circ g)^{-1}
$$

является требуемой регуляризацией бирациональной инволюции $\tau_{C}$.

ЗАмЕчаниЕ 6.6. Из конструкции отображения $\gamma$ следует, что $-K_{X_{C}}^{3}=1 / 2$.

В работе [9] был доказан следуюший результат.

ТЕорема 6.7. Пусть $Y$ - многообразие Фано с каноническими особенностями, которое бирачионально әквивалентно многообразию $X$. Тогда

$$
Y \cong\left\{\begin{array}{l}
X, \\
X_{C} \quad \text { для некоторой прямой } C \text { на } X .
\end{array}\right.
$$

Таким образом, теорема 1.11 влечет следующий результат. 
УТВЕРЖДЕНИЕ 6.8. Все конечные подгруппы группы $\operatorname{Bir}(X)$ являются подгруппами групn $\operatorname{Aut}(X)$ и $\operatorname{Aut}\left(X_{C}\right)$ для некоторой прямой $C$ на $X$.

Для каждой прямой $C$ на $X$ группа $\operatorname{Aut}\left(X_{C}\right)$ канонически содержит подгруппу $\mathbb{Z}_{2}$, которая индуцирует бирациональную инволюцию $\tau_{C}$ на многообразии $X$. Более того, можно показать, что для достаточно общего $X$

$$
\operatorname{Aut}(X) \cong \operatorname{Aut}\left(X_{C}\right) \cong \mathbb{Z}_{2}
$$

СЛЕДСТВИЕ 6.9. Для достаточно общего двойного накрытия квадрики $X$ все конечные подгруппы группы $\operatorname{Bir}(X)$ изоморфны $\mathbb{Z}_{2}$ и сопряжены подгруппам, которые порождены инволючиями $\tau_{C}$ и инволючией, индуцированной двойным накрытием $\theta$.

7. Особая трехмерная квартика. В этом пункте мы применим теорему $1.11 \mathrm{k}$ особой трехмерной квартике.

Пусть $X$ - достаточно общая квартика в $\mathbb{P}^{4}$, имеющая одну особую точку $O$ такую, что локально $O$ изоморфно обыкновенной двойной точке. Отметим, что $X$ является многообразием $\Phi$ ано с $\mathbb{Q}$ факториальными особенностями и $\operatorname{Pic}(X)=\mathbb{Z}$.

ЗАмЕчание 7.1. Квартика $X$ содержит ровно 24 различных прямых, проходящих через особую точку $O$ и группа $\operatorname{Aut}(X)$ тривиальна.

В работе [9] было показано следующее.

ТЕОрема 7.2. Для точки $O$ и каждой прямой $C$ на $X$, проходящей через особую точку $O$, существуют бирациональные перестройки

$$
\psi_{O}: X \rightarrow X_{O} \quad u \quad \psi_{C}: X \rightarrow X_{C}
$$

такие, что многообразия $X_{O}$ и $X_{C}$ являются трехмерными многообразиями Фано с каноническими особенностями, $-K_{X_{O}}^{3}=2 u-K_{X_{C}}^{3}=1 / 2$. Для кахдого трехмерного многообразия Фано $Y$ с каноническими особенностями, которое бирационально изоморфно квартике $X$, выполнено следующее:

$$
Y \cong\left\{\begin{array}{l}
X, \\
X_{O}, \\
X_{C} \quad \text { для некоторой прямой } C \text { на } X, \text { проходящей через точку } O .
\end{array}\right.
$$

Бирациональная жесткость $X$ доказана в [4] вместе со следующим результатом.

Теорема 7.3. Точка $O$ и каждая прямая $C$ наквартике $X$, проходящая через особую точку $O$, индуцируют бирациональные инволюиии $\tau_{O} u \tau_{C}$ квартики $X$ соответственно. Более того, инволюиии $\tau_{O}$ и $\tau_{C}$ порождают әруппу $\operatorname{Bir}(X)$.

Инволюции $\tau_{O}$ и $\tau_{C}$ регуляризируемы, и гипотезы 1.12 и 1.14 выполнены для квартики $X$. Из теорем 1.11 и 7.2 следует, что бирациональные инволюции $\tau_{O}$ и $\tau_{C}$ регуляризируются на многообразиях $X_{O}$ и $X_{C}$, а следующее утверждение было доказано в [9]. 
УТВЕРЖДЕНИЕ 7.4. Бирациональные отображсения $\psi_{O}$ и $\psi_{C}$ являются регуляризачией бирачиональных инволючий $\tau_{O}$ и $\tau_{C}$ соответственно.

Бирациональные отображения $\psi_{O}$ и $\psi_{C}$ можно построить аналогично тому, как была построена регуляризация бирациональньх инволюций двойного накрытия трехмерной квадрики в предыдушем пункте.

Из общности многообразия $X$ следует, что

$$
\operatorname{Aut}\left(X_{C}\right) \cong \operatorname{Aut}\left(X_{0}\right) \cong \mathbb{Z}_{2}
$$

СлЕДСТВИЕ 7.5. Все конечные подгруппы группы $\operatorname{Bir}(X)$ изоморфны $\mathbb{Z}_{2}$ и сопряжены с подгруппами, которые порождены инволюииями $\tau_{C} u \tau_{O}$.

Автор выражает свою благодарность М. Гриненко, В.А. Исковских и В.В. Шокурову за полезные беседы и В. Клейнерту, А. Радтке, М. Росчену и Л. Воцлаву за их гостеприимство во время проживания автора в Берлине.

\section{СПИСОК ЦИТИРОВАННОЙ ЛИТЕРАТУРЫ}

[1] Манин Ю. И. Кубические формы. М.: Наука, 1972.

[2] Kawamata Y., Matsuda K., Matsuki K. Introduction to the minimal model problem // Adv. Stud. Pure Math. 1987. V. 10. P. 283-360.

[3] Исковских В. А. Бирациональные автоморфизмы трехмерных алгебраических многообразий // Современные проблемы математики. 1979. Т. 13. С. 159-236.

[4] Пухликов А. В. Бирациональные автоморфизмы трехмерной квартики с простейшей особенностью // Матем. сб. 1988. Т. 135. С. 472-496.

[5] Alexeev V. General elephants of $\mathbb{Q}$-Fano 3-folds // Comp. Math. 1994. V. 91. P. 91-116.

[6] Шокуров B. B. 3-fold log models // J. Math. Sci. 1996. V. 81. P. 2667-2699.

[7] Manin Yu. I. Rational surfaces over perfect fields // Publ. Math. IHES. 1966. V. 30. P. 55-114.

[8] Манин Ю. И. Рациональные поверхности над совершенными полями II // Матем. сб. 1967. T. 72. C. $161-192$.

[9] Чельцов И.А. Антиканонические модели трехмерных многообразий Фано степени четыре // Матем. сб. 2003. Т. 194. №4. С. 147-172.

[10] Каневский Д. Структура групп, связанных с автоморфизмами кубических поверхностей // Матем. сб. 1977. Т. 103. № 2. С. 252-264.

Университет штата Джорджия, США

Поступило

E-mail: cheltsov@yahoo.com

20.01 .2003

Исправленный вариант

23.05.2003 